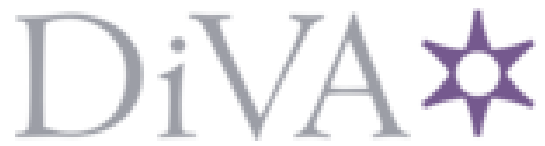

http://www.diva-portal.org

Postprint

This is the accepted version of a paper presented at HCI INTERNATIONAL 2020.

Citation for the original published paper:

Voronkov, A., Martucci, L. (2020)

Natural vs. Technical Language Preference and its Impact on Firewall Configuration In:

N.B. When citing this work, cite the original published paper.

Permanent link to this version:

http://urn.kb.se/resolve?urn=urn:nbn:se:kau:diva-76773 


\title{
Natural vs. Technical Language Preference and Their Impact on Firewall Configuration
}

\author{
Artem Voronkov and Leonardo A. Martucci \\ Karlstad University, Sweden \\ \{firstname. lastname\}@kau.se
}

\begin{abstract}
Firewalls are network security components designed to regulate incoming and outgoing traffic to protect computers and networks. The behavior of firewalls is dictated by its configuration file, which is a written sequence of rules expressed by a set of keys and parameters. In this paper, we investigate whether certain representations of firewall rule sets can affect understandability. To collect data for our investigation, we designed an online survey for an audience who are familiar with firewalls, in which we aimed to compare two different rule set representations: iptables and English. We collected data from 56 participants. Our results show that participants' perception of a certain rule set representation depends on their firewall expertise. Participants with basic or intermediate knowledge of firewalls consider rule sets expressed in English to be $40 \%$ easier to understand, whereas advanced or expert firewall users deemed it to be $27 \%$ more difficult. We will discuss the reasons for these results and describe their possible implications.
\end{abstract}

Keywords: Firewalls · Human-computer interaction - User study · Rule set representation - Survey.

\section{Introduction}

Firewalls are network security components designed to regulate incoming and outgoing traffic to protect computers and networks. To ensure that firewalls properly perform their assigned tasks, they must be correctly configured. However, people responsible for managing firewalls (administrators) often make mistakes [19], which may result in security vulnerabilities. The behavior of a firewall depends on its configuration file, which is a written sequence of rules expressed by a set of keys and parameters. The syntax is usually technical and specific to the firewall or operating system. Learning the syntax and extracting the semantics of a set of rules can be a large task for those who configure firewalls [14].

In this paper, we examine how people read and understand firewall rule sets. The goal of our study is to investigate whether representing firewall rules in a certain way can make them easier for to understand. We selected two different syntaxes that are used to describe firewall policies: 
- English, and

- iptables - a well-known Linux kernel firewall with its own policy description syntax.

To achieve our goal, we surveyed 56 people who are familiar with firewalls and collected their responses regarding understandability of the rule set representations.

Our results show a significant difference in understandability of the same rule sets expressed in iptables and English. That difference is dependent on the level of firewall expertise of the participant. The rule sets presented in English are $40 \%$ easier to understand, according to our participants with basic and intermediate knowledge of firewalls. Those more experienced in firewalls considered English rule sets to be $27 \%$ more difficult.

The rest of the paper presents a review of work related to our study in Section 2 . Our research methodology is described in Section 3. The results of our survey, discussion of our findings, and limitations of our study are presented in Section 4. Finally, the concluding remarks are given in Section 5.

\section{Related Work}

An excessive complexity of firewall languages is a well-known issue that has been studied in literature. For this reason, firewall configuration files are often of low quality as shown by Wool, who studied 84 Check Point Firewall-1 and Cisco PIX rule sets and discovered critical errors in most of them [19].

Different methods to approach this problem were suggested in scientific papers. For example, a range of higher-level firewall languages was proposed [1, 8, 20]. These languages are designed to help system administrators avoid mistakes while configuring firewalls. However, IT professionals are often reluctant to learn and utilize new languages, as Wong pointed out [18].

Others proposed different graphical user interfaces (GUI) for firewalls to improve their usability (see [13] for a survey on firewall GUIs). However, most of the proposed visualization schemes lack proper evaluation in terms of usability testing and/or user studies, as noted in [13].

In parallel to this work, we looked into usability metrics for improving firewall rule sets [15], which provide formal mathematical models to improve rule sets based on qualitative studies with IT professionals. However, such a solution was designed and tested using a single syntax (iptables).

In the field of software engineering, different programming languages were compared in terms of understandability $[4,7,16]$. Furthermore, there were some attempts to compare human and programming languages [3]. To the best of our knowledge, there has not been any research comparing different firewall languages with each other or with human languages. 


\section{Methodology}

In this section, we present our research methodology, including the details of our survey, the recruitment strategy and participants, and ethical considerations.

\subsection{Survey Details}

We collected the data through an online survey from September to December 2019. ${ }^{1}$ The survey used skip logic and therefore had a non-fixed number of questions. The participants were asked from a minimum of 12 to a maximum of 16 questions, of which four to eight were open ended. All questions were required to be answered; therefore, we added the option "not sure" in some open-ended questions, so a participant could skip them in case of uncertainty about the correctness of his or her answers.

The survey consisted of two parts: (1) we asked the participants demographicrelated questions, including age, gender, and expertise; (2) the participants were shown two firewall rule sets expressed with different syntaxes (English and iptables, in no particular order) and were asked to evaluate their understandability.

We started with two rule sets $\left\{a_{1}, b_{1}\right\}$ that were originally written using iptables syntax, one of the most common software firewalls syntaxes, and found in a public repository of real-world firewall rule sets. ${ }^{2}$ The two selected rule sets were approximately of the same complexity level, according our metrics [15]. We translated the iptables rule sets into English and obtained two new rule sets $\left\{a_{2}, b_{2}\right\}$. Each participant was shown a pair of rule sets - either $\left\{a_{1}, b_{2}\right\}$, or $\left\{a_{2}, b_{1}\right\}$, which were presented sequentially, i.e. one after the other.

To decide which pair of rule sets is to be shown, we used the following approach. We randomly selected a pair of rule sets for the first participant and stored that information together with his or her number of years of experience with configuring firewalls. When the next participant with the same experience took part in the survey, he or she was shown the other pair of rule sets. Thus, we alternated which rule set representation the participants saw first: iptables or English.

After displaying each rule set, we asked one to three open-ended questions (see an example in Figure 1) to the participants. These open-ended questions aimed to verify if they completely understood the given rule set and its functionality.

The number of questions asked to a participant depends on the correctness of his or her answers. As soon as a participant provided a correct answer, the remaining

\footnotetext{
1 The survey is available at https://survey.cs.kau.se/rulesets_comparison/

${ }^{2}$ https://github.com/diekmann/net-network
} 


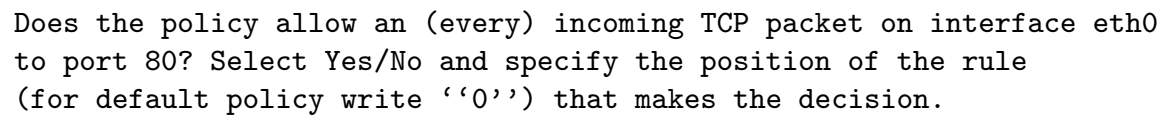

Fig. 1. An example of a question that tests whether a participant understood the given firewall rule set.

(one or two questions) were skipped. Since the questions were open ended, we considered the probability of accidental right answers (right guess) to be low.

In the last question, we asked the participants to evaluate how easy or difficult, in their opinion, to understand each of the rule sets using a 7-point Likert scale.

The survey took an average of 660 seconds $(M=579, S D=311, Q 1=400$, and $Q 3=864)$ of the participants' time to be completed.

The survey was pre-tested with five subjects before its dissemination. Although no significant changes were necessary to be implemented to the original design, the received feedback helped us eliminate some ambiguity in the wording and slightly improve the design of the study.

\subsection{Recruitment and Participants}

Reddit $^{3}$ has been demonstrated to be a good source to recruit participants, especially when people with a highly tailored knowledge are required [14]. We adopted the recruitment strategy from [14] and used the following channels for finding participants:

1. System administrators' subreddit. The Sysadmin subreddit ${ }^{4}$, which is known to be one of the largest communities of system administrators (ca. $400 \mathrm{k}$ members), yielded a significant part of our participant recruitment.

2. Other subreddits. For this study, we needed participants with varying firewall expertise. Moreover, we knew that our study would be much more time consuming, which meant that the completion rate would be significantly lower. Therefore, we reached out for three other subreddits: Networking ${ }^{5}$, Netsec $^{6}$, and Linux ${ }^{7}$, which have members who have firewall knowledge.

3. Professional networks. We contacted several colleagues from our professional networks and asked them to distribute our survey to our target group, i.e. professionals with firewall knowledge.

\footnotetext{
3 https://www.reddit.com/

${ }^{4}$ https://www.reddit.com/r/sysadmin/

${ }^{5}$ https://www.reddit.com/r/networking

${ }^{6}$ https://www.reddit.com/r/netsec

${ }^{7}$ https://www.reddit.com/r/linux
} 
Table 1. Participant demographics $(N=56)$.

\begin{tabular}{lll}
\hline & Metric & Participants \\
\hline & $18-24$ & $8(14.3 \%)$ \\
Age & $25-34$ & $27(48.2 \%)$ \\
& $35-44$ & $13(23.2 \%)$ \\
& $45-54$ & $4(7.1 \%)$ \\
& $55-64$ & $2(3.6 \%)$ \\
& Prefer not to answer & $2(3.6 \%)$ \\
Gender & & \\
& Female & $6(10.7 \%)$ \\
& Male & $43(76.8 \%)$ \\
Experience & Prefer not to answer & $6(12.5 \%)$ \\
with configuring & $4-6$ years & $7(12.5 \%)$ \\
firewalls & $<1$ year & $13(23.2 \%)$ \\
& 1-9 years & $9(16.1 \%)$ \\
Proficiency & $10+$ years & $5(9.0 \%)$ \\
with & & $22(39.2 \%)$ \\
firewalls & Basic knowledge & $11(19.6 \%)$ \\
& Intermediate & $13(23.2 \%)$ \\
& Advanced & $19(33.9 \%)$ \\
& Expert & $13(23.2 \%)$ \\
& &
\end{tabular}

The participants of our study were volunteers and received no financial compensation for taking part in the survey. Among the 516 participants who started the survey, 82 completed it (ca. $16 \%$ completion rate).

As aforementioned, we used up to three control questions to check whether a participant completely understood the given rule sets. The participants who did not give at least one correct answer for each of the two rule sets were excluded from the survey's data. Thus, 25 participants (ca. 30\% of those who finished the survey) did not meet this requirement and hence their data were discarded. Additionally, one participant was removed as he or she filled out nonsensical answers.

Table 1 summarizes the demographics of the remaining 56 participants. Our sample is skewed age- and gender-wise with $63 \%$ of participants being younger than 35 years and with only $11 \%$ of participants being female, due to the specificity of the target audience and recruitment approach. We recruited the majority of our participants via Reddit since its users are known to be younger than the general population [10]. Moreover, the percentage of female users in the selected 
subreddits is low, e.g. only $7.5 \%$ and $5.0 \%$ for the Sysadmin and Networking subreddits [2], respectively, which explains the skewness of our data sample.

As expected, the completion rate of our survey is considerably lower than that in our previous study [14] with (mainly) Reddit users $-59 \%$ and $16 \%$, respectively. There are two main reasons for such a significant difference. First, this survey consumed an average of 660 seconds, while our past study needed only an average of 177 seconds to be completed. Since all our participants are volunteers, a significant number of participants dropped out from our survey. Second, the control questions in the survey, which tested the participants' understanding of the given firewall rule sets, significantly increased the dropout rate, as we could evaluate in our results. Such control questions were not included in our past study [14].

\subsection{Ethical Considerations}

The survey was conducted in accordance with the Swedish Ethical Review Act [11] and the Good Research Practice guidelines from the Swedish Research Council [12]. Prior to data collection, this study was approved by our institutional ethics review board (IRB). The following precautions were considered to ensure that the participants were treated ethically and with respect:

- The participants completed an IRB-approved consent form before starting the survey. The purpose of the study, its approximate duration, our commitment to confidentiality, and their rights as participants, including the right to withdraw from the study at any point in time, were stated in the form.

- The minimum amount of personal data (see Table 1) was collected.

- No sensitive personal data were collected.

\section{Results and Discussion}

From each participant, we obtained two difficulty scores: one for the rule set expressed using iptables and one for the rule set expressed in English. Since our samples are dependent, the data were analyzed using the Wilcoxon signed-rank test [17] to compare the average scores of these samples and assess them for significant differences. The Wilcoxon signed-rank test is a non-parametric equivalent to the paired sample t-test that does not carry assumptions of normality of data distribution and can be applied to ordinal data [6].

The Wilcoxon test did not yield significant results when all data points were considered. $(p=0.843)$. However, a deeper look at the data shows that the data records differ significantly depending on the participant's firewall expertise. We then divided our data set into two parts:

1. 24 participants who have basic or intermediate knowledge on firewalls 
Table 2. Descriptive statistics for the subset of participants with basic or intermediate firewall knowledge.

\begin{tabular}{lccccc}
\hline & N & Mean & Std. Deviation & Minimum & Maximum \\
\hline Iptables_Score & 24 & 2.92 & 1.248 & 1 & 5 \\
English_Score & 24 & 4.08 & 1.381 & 1 & 7 \\
\hline
\end{tabular}

Table 3. The Wilcoxon signed-rank test for the subset of participants with basic or intermediate firewall knowledge.

\begin{tabular}{llccc}
\hline & & N & Mean Rank & Sum of Ranks \\
\hline English_Score - & Negative Ranks & $5^{\mathrm{a}}$ & 6.20 & 31.00 \\
Iptables_Score & Positive Ranks & $15^{\mathrm{b}}$ & 11.93 & 179.00 \\
& Ties & $4^{\mathrm{c}}$ & & \\
& Total & 24 & & \\
\hline
\end{tabular}
a. English_Score $<$ Iptables_Score
b. English_Score $>$ Iptables_Score
c. English_Score = Iptables_Score

2. 32 participants who are advanced or expert firewall users

For each of these two data subsets, we displayed some descriptive statistics and ran the Wilcoxon signed-rank test (see Tables $2-5$ ).

From Tables 2 and 4, we see that mean scores for iptables and English rule sets differ: 2.92 and 4.08 for less-experienced participants and 4.66 and 3.66 for proficient participants. The Wilcoxon signed-rank test indicated that the difficulty score of the rule sets expressed in English was statistically significant higher than the score of the same rule sets when presented as iptables $(z=2.823$, $p=0.005)$ for the subset with a limited firewall knowledge; i.e. the rule sets in English were more easily understood by the participants with basic or intermediate knowledge on firewalls. On the contrary, advanced and expert firewall users considered the iptables rule sets to be significantly easier than the corresponding English ones $(z=2.350, p=0.019)$.

Additionally, we measured the effect size of the Wilcoxon test for both of the data subsets. We calculated Pearson's correlation coefficient, $r$, as the following [9]:

$$
r=\frac{z}{\sqrt{N}} \quad \text { where } \mathrm{N} \text { corresponds to the total number of participants. }
$$

For the first and second subsets, $r$ equals to 0.47 and 0.42 , respectively. Both $r$ lie between 0.3 and 0.5 , which corresponds to moderate effect [5]. 
Table 4. Descriptive statistics for the subset of participants with advanced or expert firewall knowledge.

\begin{tabular}{lclccc}
\hline & N & Mean & Std. Deviation & Minimum & Maximum \\
\hline Iptables_Score & 32 & 4.66 & 1.638 & 1 & 7 \\
English_Score & 32 & 3.66 & 1.734 & 1 & 7 \\
\hline
\end{tabular}

Table 5. The Wilcoxon signed-rank test for the subset of participants with advanced or expert firewall knowledge.

\begin{tabular}{llccc}
\hline & N & Mean Rank & Sum of Ranks \\
\hline English_Score - & Negative Ranks & $20^{\mathrm{a}}$ & 15.25 & 305.00 \\
Iptables_Score & Positive Ranks & $8^{\mathrm{b}}$ & 12.63 & 101.00 \\
& Ties & $4^{\mathrm{c}}$ & & \\
& Total & 32 & & \\
\hline
\end{tabular}
a. English_Score $<$ Iptables_Score
b. English_Score $>$ Iptables_Score
c. English_Score =Iptables_Score

\subsection{Discussion}

A possible explanation for why our participants prefer a certain representation of rule sets depending on their firewall expertise is that learning the syntax of iptables requires a significant effort.

Inexperienced system administrators and other users with basic or intermediate knowledge on firewalls considered iptables rule sets difficult to understand, since they are usually not extensively familiar with its syntax. Therefore, the rule sets that are expressed in English $\{a 2, b 2\}$ were easier for them. In contrast, the participants with extensive experience on firewalls are more familiar with the iptables syntax, which is more concise than English and therefore preferred by them.

Different representations can be used to show firewall rule sets to different categories of people. In general, system administrators have many other responsibilities apart from configuring firewalls [14], and they do not usually have time to learn multiple and complex firewall syntaxes. Moreover, inexperienced users can be trained using a language that does not require a significant time investment to be learned. In particular, rule sets expressed in English are suitable, since there is no additional learning of syntax involved. Furthermore, if several people with different firewall expertise work on the same rule set, a translation tool that can convert it from one representation to another would be extremely valuable. 


\subsection{Limitations}

One of the limitations of our survey is that most of the participants were recruited through Reddit, an online platform. Since the study participants were volunteers, there is a self-selection bias that leads to the sample not being fully representative. Moreover, since they did not receive any financial compensation, and the survey consumed more than 10 minutes of their time on average to be answered, its completion rate was rather low, which in turn significantly reduced the sample size of our survey.

The study was conducted online and hence we could not observe the participants answering the questions. Although we included (up to three) control questions to improve the quality of the survey by selecting only participants with some experience in dealing with firewall rule sets, the answers to some questions, e.g. demographic related, might still be untrue. There is also the possibility of misinterpreted or misunderstood questions by the participants. We mitigated this limitation by carefully considering the design of the survey and pretesting our survey with five people.

The syntax of iptables is not very expressive while being considerably verbose. It was recommended by some Reddit users to utilize Cisco Adaptive Security Appliance (ASA) configuration files that are familiar to a wider audience and thereby increase the number of potential participants. ${ }^{8}$ The translation of firewall rule sets into English might also be improved and eventually automated.

\section{Conclusion}

In this paper, we presented an online study for an audience who are familiar with firewalls, in which we compared two different rule set representations. The survey was successfully completed by 56 participants and their data were analyzed using the Wilcoxon signed-rank test.

Our results show that a rule set expressed in English received on average a $40 \%$ higher difficulty score (easier to understand) from inexperienced users (with basic and intermediate knowledge on firewalls) when compared to the corresponding iptables rule set.

Among the participants who are advanced or expert firewall users, we observed the opposite. The rule sets presented in the form of iptables were $27 \%$ easier to read and understand when compared to the corresponding ones in English.

Our work demonstrates that users might prefer different rule set representations depending on their firewall expertise. The theoretical significance of this work is

\footnotetext{
${ }^{8}$ https://www.cisco.com/c/en/us/products/security/adaptive-security-applianceasa-software/index.html
} 
that we showed the need for multiple rule set representations, so both inexperienced users and experts could efficiently use the firewall. This finding presents opportunities for future research. For example, manufacturers of firewalls might need to compare several rule set representations to determine their strengths and be able to create a better firewall interface.

As a part of our future work, we plan to increase the number of rule set representations tested as well as to recruit more participants for future surveys.

Acknowledgments We are very grateful to everyone who participated in our survey. We would also like to thank the moderators of the Sysadmin ( $r$ /sysadmin), Networking ( $\mathrm{r} /$ networking), Netsec ( $\mathrm{r} / \mathrm{netsec})$, Linux ( $\mathrm{r} / \mathrm{linux}$ ) subreddits for allowing us to reach out to their community.

This work was supported by the Knowledge Foundation of Sweden HITS project and by the Swedish Foundation for Strategic Research SURPRISE project.

\section{References}

1. Bodei, C., Degano, P., Galletta, L., Focardi, R., Tempesta, M., Veronese, L.: Language-independent synthesis of firewall policies. In: 2018 IEEE European Symposium on Security and Privacy (EuroS\&P). pp. 92-106. IEEE (2018)

2. Burkhart, B.: Subreddit gender ratios. http://bburky.com/subredditgenderratios/ (2017)

3. Connolly, J.H.: Context in the study of human languages and computer programming languages: A comparison. In: International and Interdisciplinary Conference on Modeling and using Context. pp. 116-128. Springer (2001)

4. Crosby, M.E., Scholtz, J., Wiedenbeck, S.: The roles beacons play in comprehension for novice and expert programmers. In: PPIG. p. 5 (2002)

5. Field, A.: Discovering statistics using IBM SPSS statistics. sage (2013)

6. Meek, G.E., Ozgur, C., Dunning, K.: Comparison of the t vs. wilcoxon signed-rank test for likert scale data and small samples. Journal of modern applied statistical methods 6(1), 10 (2007)

7. Nanz, S., Torshizi, F., Pedroni, M., Meyer, B.: Design of an empirical study for comparing the usability of concurrent programming languages. Information and Software Technology 55(7), 1304-1315 (2013)

8. Pozo, S., Varela-Vaca, A., Gasca, R.: Afpl2, an abstract language for firewall acls with nat support. In: 2009 Second International Conference on Dependability. pp. 52-59. IEEE (2009)

9. Rosenthal, R., Cooper, H., Hedges, L.: Parametric measures of effect size. The handbook of research synthesis 621, 231-244 (1994)

10. Sattelberg, W.: The demographics of reddit: Who uses the site? https://www.techjunkie.com/demographics-reddit/ (2018)

11. Svensk Författningssamling (SFS): Lag (2003:460) om etikprövning av forskning som avser människor [The Act concerning the Ethical Review of Research Involving Humans]. Utbildningsdepartementet, Stockholm, Sweden (2003) 
12. Swedish Research Council (VR): Conducting ethical research. https://www.vr.se/ (2018), accessed: 2019-12-12

13. Voronkov, A., Iwaya, L.H., Martucci, L.A., Lindskog, S.: Systematic literature review on usability of firewall configuration. ACM Comput. Surv. 50(6) (Dec 2017). https://doi.org/10.1145/3130876

14. Voronkov, A., Martucci, L.A., Lindskog, S.: System administrators prefer command line interfaces, don't they? an exploratory study of firewall interfaces. In: Fifteenth Symposium on Usable Privacy and Security (SOUPS 2019) (2019)

15. Voronkov, A., Martucci, L.A., Lindskog, S.: Measuring the usability of firewall rule sets. IEEE Access, To appear (2020)

16. Wiedenbeck, S., Ramalingam, V., Sarasamma, S., Corritore, C.L.: A comparison of the comprehension of object-oriented and procedural programs by novice programmers. Interacting with Computers 11(3), 255-282 (1999)

17. Wilcoxon, F.: Individual comparisons by ranking methods. Biometrics Bulletin 1(6), 80-83 (1945), http://www.jstor.org/stable/3001968

18. Wong, T.: On the usability of firewall configuration. In: Symposium on usable privacy and security (2008)

19. Wool, A.: Trends in firewall configuration errors: Measuring the holes in swiss cheese. IEEE Internet Computing 14(4) (2010)

20. Zhang, B., Al-Shaer, E., Jagadeesan, R., Riely, J., Pitcher, C.: Specifications of a high-level conflict-free firewall policy language for multi-domain networks. In: Proceedings of the 12th ACM symposium on Access control models and technologies. pp. 185-194. ACM (2007) 